\title{
Uropathogenic Escherichia coli strain CFT073 disrupts NLRP3 inflammasome activation
}

\author{
Anna Waldhuber, ${ }^{1}$ Manoj Puthia, ${ }^{2}$ Andreas Wieser, ${ }^{3}$ Christine Cirl, ${ }^{1}$ Susanne Dürr, ${ }^{1}$ Silke Neumann-Pfeifer, ${ }^{4}$ Simone Albrecht, ${ }^{4}$ \\ Franziska Römmler, ${ }^{1}$ Tina Müller, ${ }^{1}$ Yunji Zheng, ${ }^{2}$ Sören Schubert, ${ }^{3}$ Olaf Groß, ${ }^{5}$ Catharina Svanborg, ${ }^{2}$ and Thomas Miethke ${ }^{4}$ \\ IInstitut für Medizinische Mikrobiologie, Immunologie und Hygiene, Technische Universität München, Munich, Germany. ${ }^{2}$ Department of Microbiology, Immunology and Glycobiology (MIG), \\ Institute of Laboratory Medicine, Lund University, Lund, Sweden. ${ }^{3}$ Max von Pettenkofer-Institut für Hygiene und Medizinische Mikrobiologie, Ludwig-Maximilians-Universität, \\ Munich, Germany. ${ }^{4}$ Institute of Medical Microbiology and Hygiene, University of Heidelberg, Mannheim, Germany. ${ }^{5}$ Institut für Klinische Chemie und Pathobiochemie am \\ Klinikum Rechts der Isar der Technischen Universität München, Munich, Germany.
}

\begin{abstract}
Successful bacterial pathogens produce an array of virulence factors that allow subversion of the immune system and persistence within the host. For example, uropathogenic Escherichia coli strains, such as CFT073, express Toll/ IL-1 receptor-containing (TIR-containing) protein C (TcpC), which impairs TLR signaling, thereby suppressing innate immunity in the urinary tract and enhancing persistence in the kidneys. Here, we have reported that TcpC also reduces secretion of IL-1 $\beta$ by directly interacting with the NACHT leucin-rich repeat PYD protein 3 (NLRP3) inflammasome, which is crucial for recognition of pathogens within the cytosol. At a low MOI, IL-1ß secretion was minimal in CFT073-infected macrophages; however, IL-1 $\beta$ release was markedly increased in macrophages infected with CFT073 lacking tcpC. Induction of IL-1及 secretion by CFT073 and tcpC-deficient CFT073 required the NLRP3 inflammasome. TcpC attenuated activation of the NLRP3 inflammasome by binding both NLRP3 and caspase-1 and thereby preventing processing and activation of caspase-1. Moreover, in a murine urinary tract infection model, CFT073 infection rapidly induced expression of the NLRP3 inflammasome in the bladder mucosa; however, the presence of TcpC in WT CFT073 reduced IL-1 $\beta$ levels in the urine of infected mice. Together, these findings illustrate how uropathogenic $E$. coli use the multifunctional virulence factor TcpC to attenuate innate immune responses in the urinary tract.
\end{abstract}

\section{Introduction}

Urinary tract infections (UTIs) are among the most common human infections (1) and are a frequent cause of antibiotic therapy. In view of increasing resistance rates against antibiotics, treatment failures become an important issue. In the search for new approaches to treating these infections, the complex interactions of bacterial virulence factors with their host targets need to be deciphered. Bacterial virulence factors are crucial for adherence to host cells, improvement of nutrition of the pathogen, mediation of motility, and manipulation of the inflammatory response of the host. In the case of UTIs, uropathogenic Escherichia coli (UPEC) strains are the predominant cause. They are equipped with a variety of virulence factors including fimbrial adhesins, toxins, invasins, autotransporter proteins, iron-acquisition systems, and flagella (2-4), together enabling the bacteria to attack host tissues and successfully establish the infection.

In addition, bacteria survive by directly manipulating the antibacterial host defense. We discovered a new type of virulence factor in UPECs that attenuated the inflammatory host response and promoted bacterial survival in infected tissues (5). The Toll/IL-1 receptor-containing (TIR-containing) protein $\mathrm{C}(\mathrm{TcpC})$ contained a TIR domain and impaired the TLR signaling cascade by binding to the adapter molecule myeloid differentiation factor 88 (MyD88)

Conflict of interest: The authors have declared that no conflict of interest exists. Submitted: March 24, 2015; Accepted: April 7, 2016.

Reference information: J Clin Invest. 2016;126(7):2425-2436. doi:10.1172/JCI81916.
(5), which controls signaling of all TLRs except TLR3. The binding site of TcpC to MyD88 was precisely mapped and comprised the CD, DE, and EE loops of MyD88 (6). Aside from MyD88, TcpC also bound to TLR4, thereby attenuating the MyD88-independent arm of TLR4 signaling (6). Molecules related to TcpC have now been found in Brucella spp. and Salmonella enterica subspecies enterica serovar Enteritidis as well as in non- or less pathogenic bacteria, such as Paracoccus denitrificans (5, 7-9). We showed previously that TcpB from Brucella spp. also bound to MyD88 (5), elucidated its crystal structure, and confirmed its interaction with the TIR-associated protein (TIRAP) (10). Thus, these proteins evolved to interfere with several components of the TLR system.

Relevance of TcpC to acting as virulence factor in vivo was analyzed by infecting mice with the UPEC strain CFT073, which harbors the $t c p C$ gene on a pathogenicity island ( $\operatorname{ser} U$ island) (11, 12). The results showed that CFT073 replicated to higher numbers in urine and kidneys compared with a tcpC-deficient CFT073 strain. Moreover, the WT, but not the $t c p C$-deficient, isogenic mutant caused kidney abscesses, demonstrating the potency of this new virulence factor (5). Children as well as adults suffering from pyelonephritis harbor $t c p C^{+}$UPEC strains in $40 \%$ or $50 \%$ of cases, respectively $(5,12)$. Interestingly, $t c p C^{+}$strains were exclusively found in the phylogenetic group B2, and the gene is strongly associated with the high pathogenicity island (12).

Interaction of TcpC with MyD88 reduced the secretion of proinflammatory cytokines such as TNF during an infection of macrophages with CFT073, and the recombinant protein sup- 

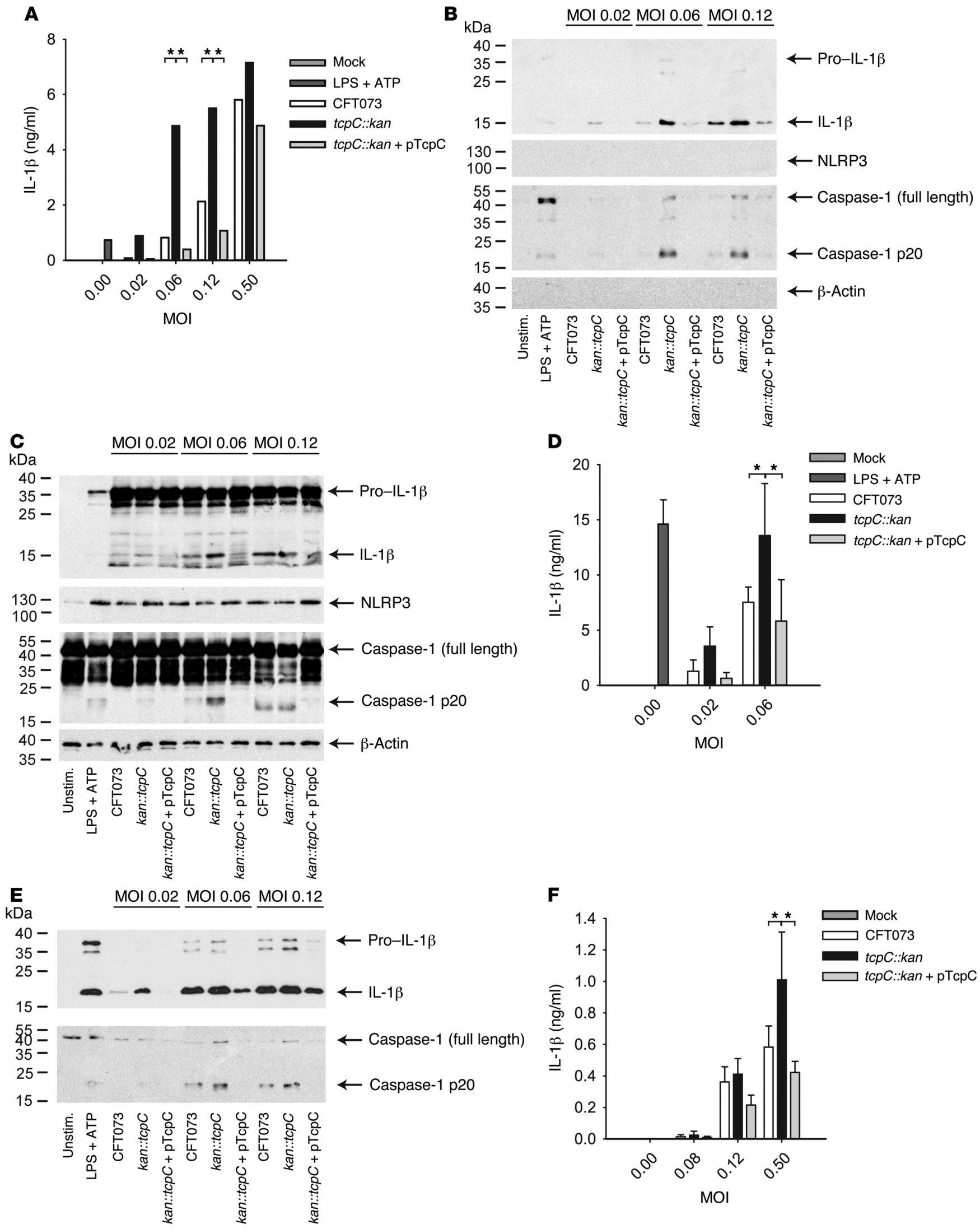
Figure 1. TcpC-mediated reduction of IL-1 $\beta$ secretion by innate immune cells upon infection with CFT073. We infected BMDMs (A-C), BMDCs ( $\mathbf{D}$ and $\mathbf{E}$ ), and HCV29 cells (F) with titrated amounts of CFTO73, the tcpC-deficient strain CFTO73 tcpC::kan, or the tcpC-complemented strain CFT073 tcpC::kan+pTcpC, as indicated. IL-1 $\beta$ was quantified in the culture supernatants after 3 hours of culture by ELISA (A, D, and F). Stimulation of cells with ATP plus LPS served as positive and the culture of cells in the absence of bacteria as negative control. Data represent mean of 2 (A), mean \pm SD of 8 (D), and mean \pm SD of 4 (F) individual cultures. A depicts a representative experiment out of 4 . Culture supernatants (B and $\mathbf{E}$ ) and the cytosol of host cells (C) were analyzed by Western blot to detect mature IL-1 $\beta$, processed caspase-1, and NLRP3. $\beta$-actin served as loading control. ${ }^{*} P<0.05$, 1-way ANOVA, post hoc Bonferroni's multiple comparison test.

pressed TNF secretion induced by a variety of pathogen-associated molecular patterns (PAMPs), indicating its potential as an antiinflammatory agent $(5,13)$.

UPEC infections cause inflammation via TLR4. Both MIP-2 and PMNs were elevated in the urine of WT mice during UTI, but these responses to infection were suppressed in TLR4-deficient mice (13). Similar results were observed with MyD88-deficient animals. Thus, UPEC-induced inflammation in the urinary tract is triggered via the TLR4/MyD88 signaling pathway.

During acute infections, UPECs form intracellular bacterial communities (IBCs) via type 1 fimbriae in the cytosol of bladder epithelial cells (14). UPEC strains that were defective in IBC formation were attenuated and cleared from the urinary tract (15). IBCs and other intracellular pathogens are presumably not recognized by the TLR system, since TLRs are either expressed at the cell membrane or at endosomal membranes. Instead, the family of nucleotide-binding oligomerization domain (NOD) proteins is crucial to detecting pathogens in the cytosol of host cells (16). The NACHT leucin-rich repeat PYD protein 3 (NLRP3), which belongs to 1 of 3 subfamilies of NOD proteins, recognizes different bacteria, such as Staphylococcus aureus and Listeria monocytogenes, and PAMPs, such as pore-forming toxins, but also extracellular ATP and monosodium urate crystals (16). Upon interaction with the adapter protein apoptosis-associated speck-like protein (ASC) and subsequent oligomerization of caspase-1, the latter is autocatalytically activated. Active caspase- 1 in turn cleaves IL-1 $\beta$, thereby maturing pro-IL-1 $\beta$ to its biologically active form. Both cleaved caspase- 1 and mature IL-1 $\beta$ are then secreted. As the NLRP3 inflammasome senses a variety of PAMPs and bacteria, including UPEC strains (17), we hypothesized that UPECs might attenuate this pattern-recognition system to reduce IL-1 $\beta$ secretion.

\section{Results}

TcpC impairs $I L-1 \beta$ release by host cells. Secretion of mature IL-1 $\beta$ requires the presence of pro-IL-1 $\beta$, which is produced TLR dependently. Since TcpC bound to MyD88 and TLR4 and impaired the TLR signaling cascade, we initially speculated that TcpC might influence the secretion of IL- $1 \beta$ by reducing pro-IL- $1 \beta$ (5). Infection of BM-derived macrophages (BMDMs) with CFT073 revealed that the microorganism induced the release of IL-1 $\beta$ (Figure 1A). Interestingly, the isogenic tcp C-mutant CFT073 tcpC::kan strain induced higher levels of IL-1 $\beta$ in the culture supernatant in comparison with the WT strain (Figure 1A). Complementation of the $t c p C$-mutant strain CFT073 tcpC::kan with a $t c p C$-containing plasmid (pTcpC) decreased IL-1 $\beta$ secretion to the level of the WT strain (Figure 1A). The difference between the strains was less pronounced at an MOI of 0.5. Analysis of the culture supernatant by Western blot revealed that the mature form of IL-1 $\beta$ was present (Figure 1B). We also found that the cleavage product $\mathrm{p} 20$ of caspase- 1 was released during the infection, and this was likewise considerably reduced by TcpC. The absence of NLRP3 and $\beta$-actin, but also lactate dehydrogenase (LDH), in the supernatant indicated that host cells remained intact during infection (Figure 1B and Supplemental Figure 1; supplemental material available online with this article; doi:10.1172/JCI81916DS1).

Reduced processing of IL- $1 \beta$ and caspase- 1 by TcpC was also detected in the cytosol of infected cells. Remarkably, TcpC did not influence cellular pro-IL-1 $\beta$ levels (Figure $1 C$ ). In contrast to our expectation, the expression of pro-IL-1 $\beta$ was strongly induced by all 3 CFT073 strains (Figure 1C and Supplemental Figure 2). This finding may be explained by the complex regulation of the IL1B gene. Thus, the endotoxin-induced expression of pro-IL-1 $\beta$

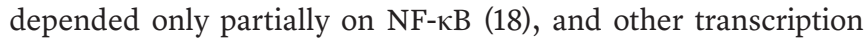
factors, such as phosphorylated IRF8 and nonphosphorylated STAT1, were critical (19). We also observed that unstimulated BMDMs expressed full-length caspase-1, whereas NLRP3 expression required stimulation with LPS or infection with CFT073 strains (Figure 1C). Infection of BM-derived DCs (BMDCs) with low MOIs of WT CFT073, CFT073 tcpC::kan, or CFT073 $t c p C:: k a n+p T c p C$ confirmed that the presence of the $t c p C$ gene reduced the secretion of mature IL- $1 \beta$ and cleaved caspase- 1 into the culture supernatant (Figure 1, D and E). These results were also reproduced upon infection of the human uroepithelial cell line HCV29 with all 3 variants of CFT073 (Figure 1F).

These findings suggested that CFT073 induced the expression and function of the NLRP3 inflammasome and that TcpC dampened caspase- 1 cleavage and release of mature IL-1 $\beta$. We then determined whether the expression of the NLRP3 inflammasome is similarly induced during a bladder infection with CFT073 in vivo. We found that uninfected bladder epithelial cells only weakly expressed ASC, NLRP3, or IL-1 $\beta$ (Figure 2, A-D). Intriguingly, CFT073 and its tcpCdeficient mutant CFT073 tcpC::kan strongly induced the expression of ASC, NLRP3, and pro-IL-1 $\beta$ or mature IL-1 $\beta$ preferentially at the apical part of the bladder epithelium (Figure 2, A-D). These findings indicated that the NLRP3 inflammasome may fulfill an important function in vivo during an acute infection of the bladder.

The NLRP3 inflammasome senses CFTO73 and TcpC suppresses its activity. We subsequently analyzed the role of caspase- 1 in CFT073-induced IL-1 $\beta$ release using caspase-1/11-deficient BMDMs (20). In response to the WT strain, release of IL-1 $\beta$ was completely dependent on caspase-1/11 and the presence of the $t c p C$ gene again correlated with a reduction of IL-1 $\beta$ secretion by WT cells (Figure 2E). Treating BMDMs with the caspase- 1 inhibitor Z-YVAD-FMK, which impairs caspase-1, also reduced IL-1 $\beta$ release induced by CFT073 or the mutant strain CFT073 tcpC::kan (Figure 2F). Furthermore, IL-1 $\beta$ release was prevented in ASC-deficient or NLRP3-deficient BMDCs upon infection with CFT073, the mutant strain CFT073 tcpC::kan, or the complemented mutant CFT073 tcpC::kan+pTcpC (Figure 2G). Taken together, the results suggested that CFT073 was sensed by the NLRP3 inflammasome and its activity was suppressed by TcpC. 
A

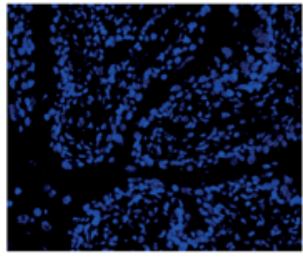

B

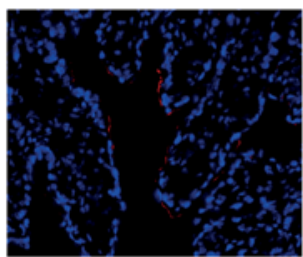

C

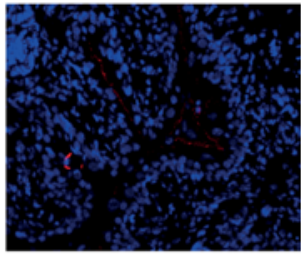

D

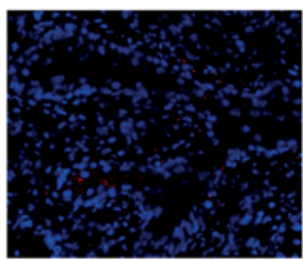

Uninfected
Bacteria/neutrophils/nuclei

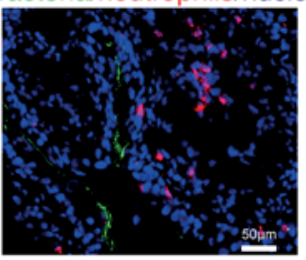

ASC/nucle

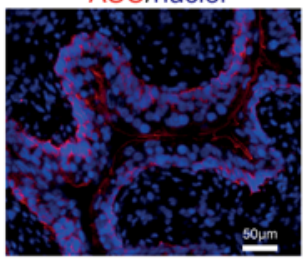

NLRP3/nuclei

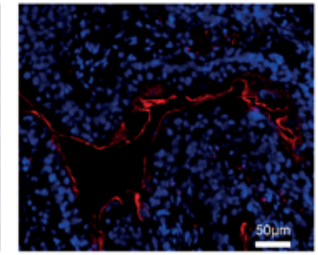

Pro + mature IL-1 $\beta /$ nuclei

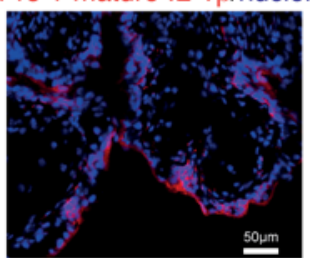

CFT073

$\mathbf{F}$

E

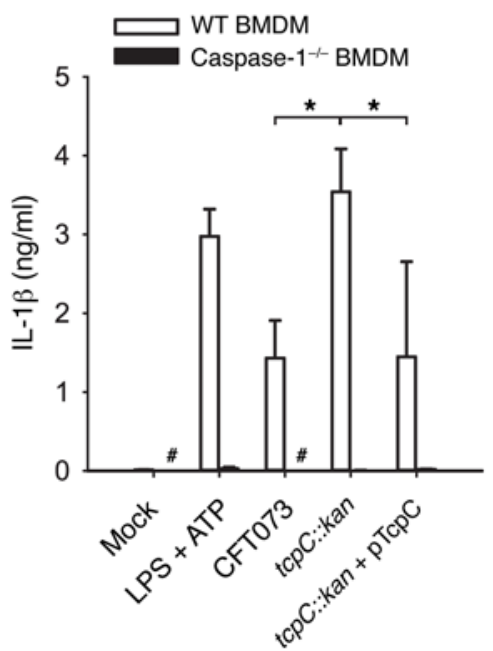

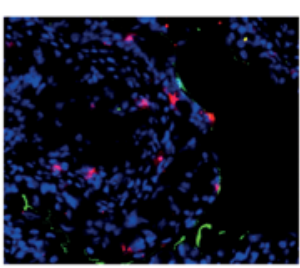
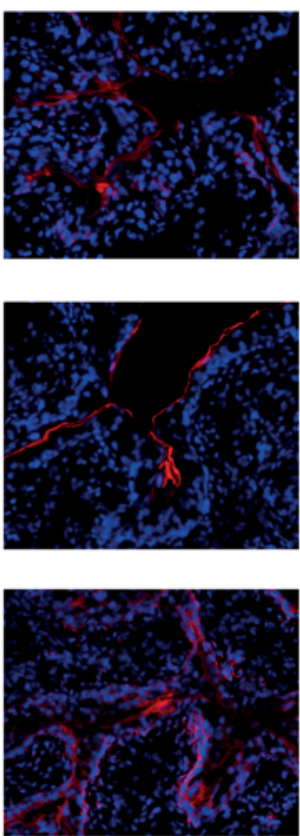

CFT073 tcpC::kan

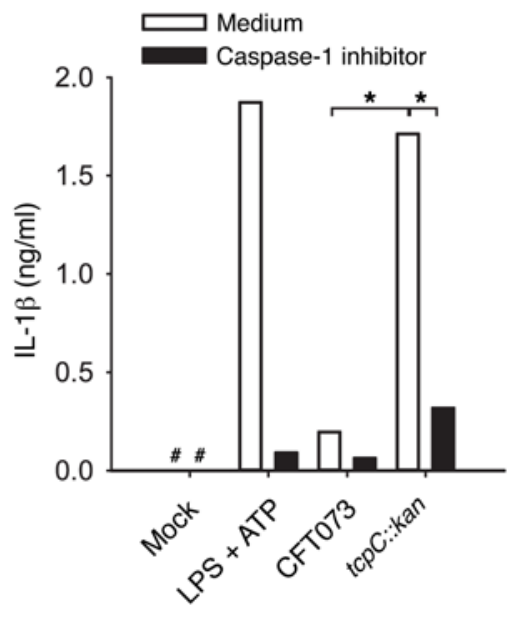

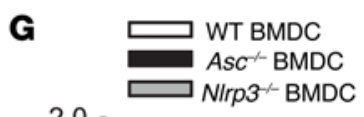

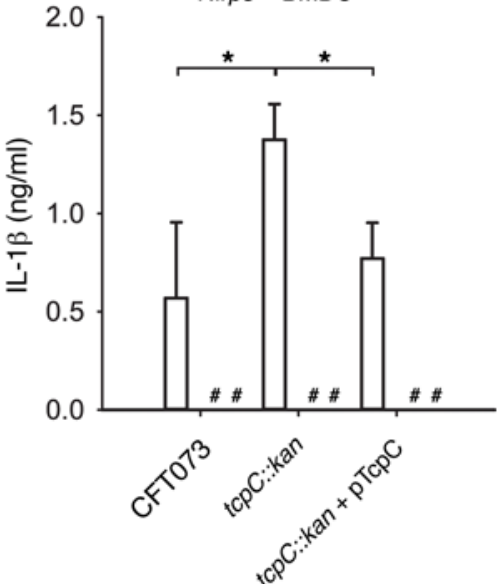

Figure 2. CFT073 induced the expression of the NLRP3 inflammasome in vivo and triggered IL-1 $\beta$ secretion via this inflammasome. We infected C57BL/6 mice with CFT073 ( $n=5$ mice) or CFT073 tcpC::kan ( $n=5$ mice) at $10^{8} \mathrm{CFU} /$ mouse. Uninfected mice $(n=3)$ served as controls. We prepared bladder sections 24 hours after infection and stained nuclei of the bladder mucosa together with bacteria and neutrophils (A), ASC (B), NLRP3 (C), or IL-1 $\beta$ (D). Representative views are shown. WT or caspase-1/11-deficient BMDMs were first stimulated with LPS for 3 hours and then infected (MOI 0.02) with CFT073, the tcpC-deficient strain CFT073 tcpC::kan, or the tcpC-complemented strain CFT073 tcpC::kan+pTcpC (E). BMDCs were infected with CFT073 or CFT073 tcpC::kan (MOI 0.1) in the presence or absence of the caspase-1 inhibitor Z-YVAD-FMK (F). Mock-treated cells served as negative and LPS plus ATPtreated cells as positive control. (C) We infected BMDCs (MOI 0.01) from WT, ASC-deficient, or NLRP3-deficient mice with CFT073, CFT073 tcpC::kan, or CFT073 tсpC::kan+pTcpC. IL-1 $\beta$ was determined in the culture supernatant after 3 hours of culture. Data represent mean \pm SD of 3 (E and $\mathbf{C}$ ) or mean of 2 (F) individual cultures. Note that in all experiments, TcpC attenuated the IL-1 $\beta$ response of WT cells. "Below detection limit; ${ }^{*} P<0.05$, 1-way ANOVA, post hoc Bonferroni's multiple comparison test. 
A

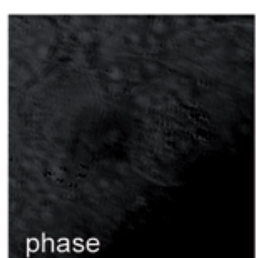

C

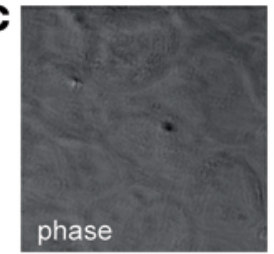

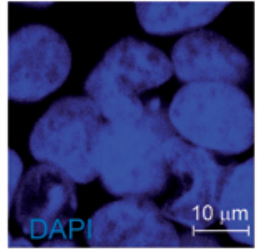
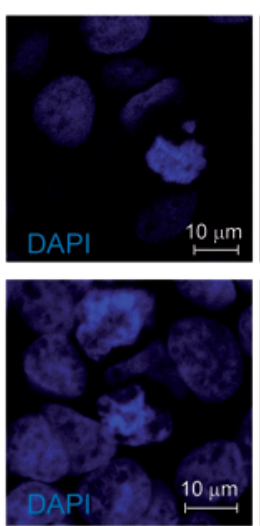
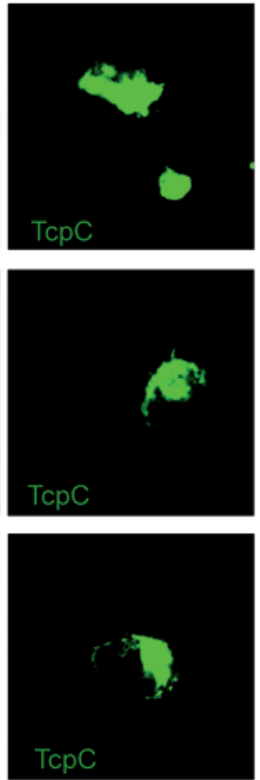
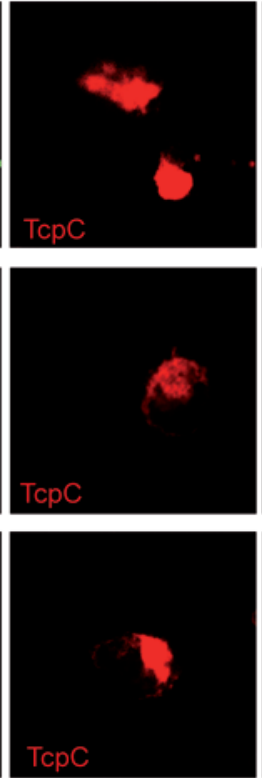
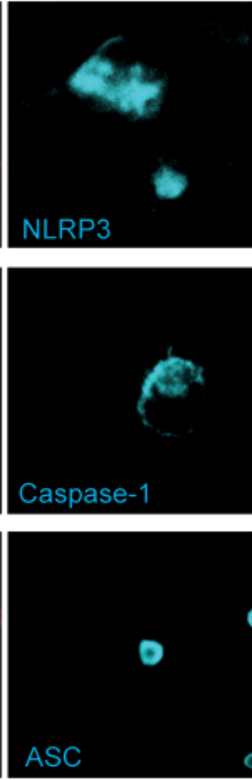
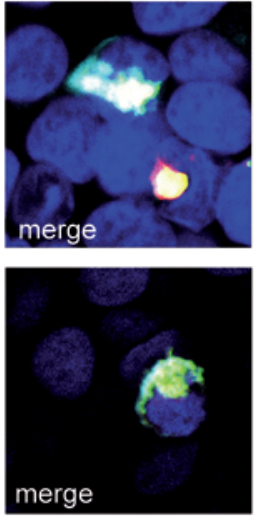

merge

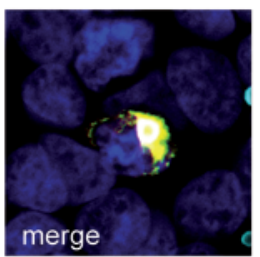

\section{D}

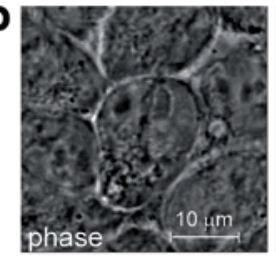

E

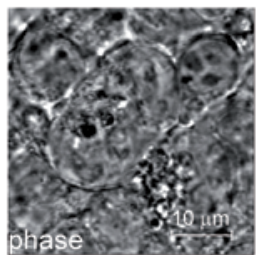

$\mathbf{F}$

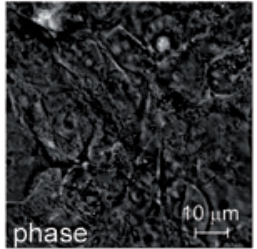

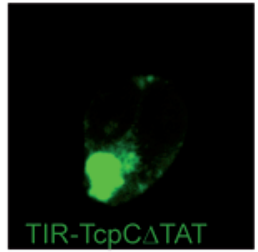
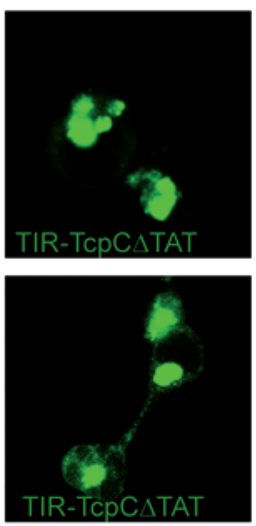
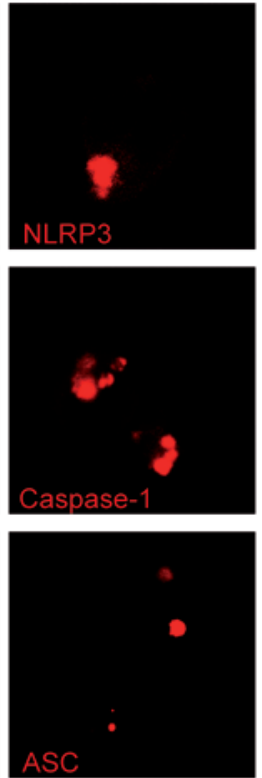
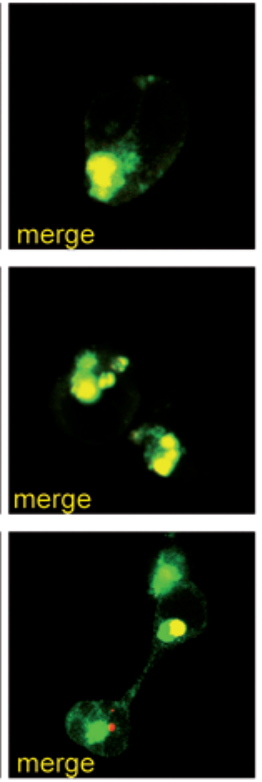

Figure 3. TcpC colocalized with NLRP3 and caspase-1. We transfected HEK293 cells with plasmids encoding EGFP/DsRed double-labeled TcpC together with either FLAG-tagged NLRP3 (A), FLAG-tagged caspase-1 (B), or FLAG-tagged ASC (C). FLAG-tagged proteins were visualized using a mouse anti-FLAC followed by an antimouse Alexa Fluor 633-labeled antibody. Cell nuclei were stained with DAPI. Confocal microscopy shows cell nuclei in blue, TcpC in green and red, and NLRP3, caspase-1, and ASC in turquoise. We also transfected HEK293 cells with EGFP-labeled TIR-TcpC $\triangle T A T$ together with DsRed-labeled NLRP3 (D), DsRed-labeled caspase-1 (E), or DsRed-labeled ASC (F). To avoid possible fixation artifacts, cells were not fixed. HEK293 cells were transfected with plasmids encoding EGFP-labeled ASC (C) or EGFPlabeled TIR-TcpC $\triangle$ TAT $(\mathbf{H})$ together with DsRed-labeled NLRP3 and FLAG-tagged caspase-1. FLAG-tagged caspase-1 was visualized using a mouse anti-FLAC followed by an anti-mouse Alexa Fluor 633-labeled antibody. Cell nuclei were stained with DAPI. Confocal microscopy shows cell nuclei in blue, ASC and TIR-TcpC $\triangle T A T$ in green, NLRP3 in red, and caspase-1 in turquoise.
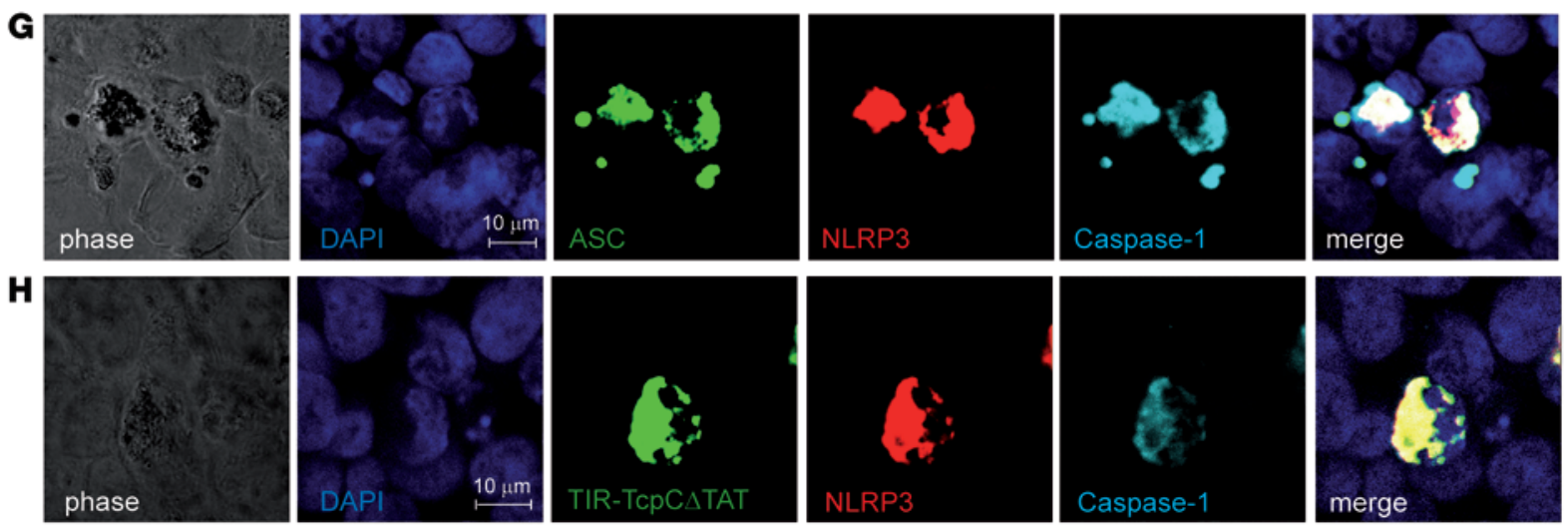

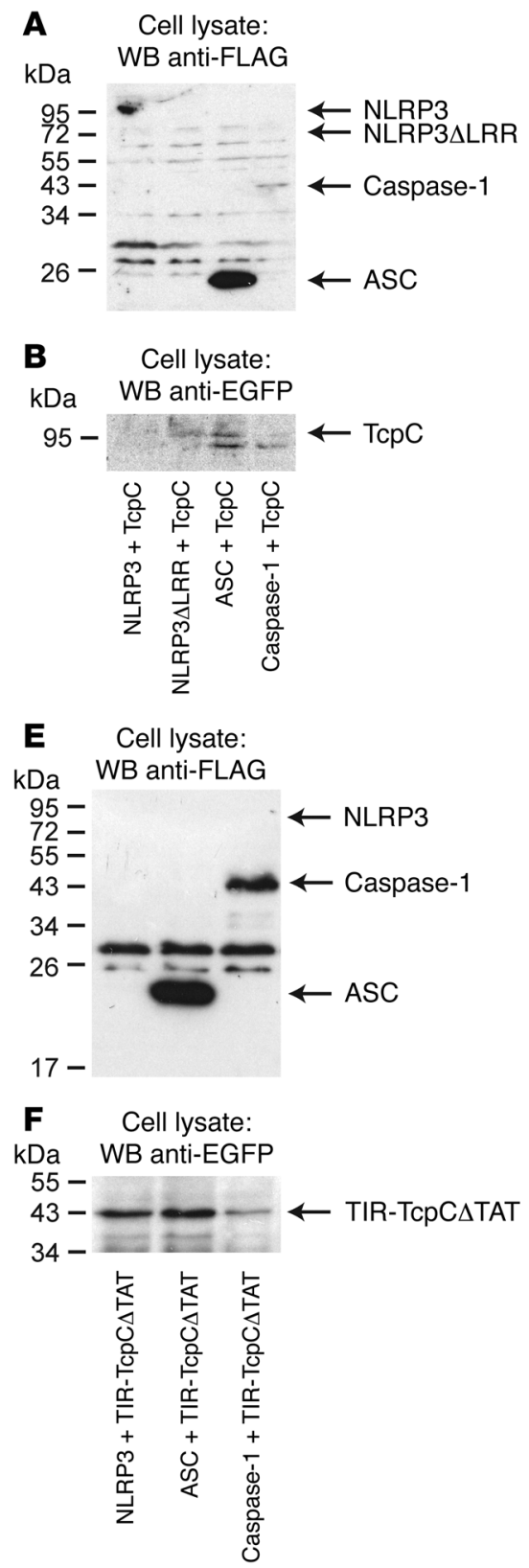

$\begin{array}{cc}\text { C } & \text { IP anti-FLAG } \\ \mathrm{kDa} & \text { WB anti-FLAG }\end{array}$

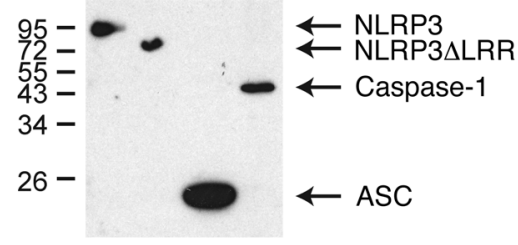

$\begin{array}{ll}\text { D } & \text { IP anti-FLAG } \\ \text { WB anti-EGFP }\end{array}$

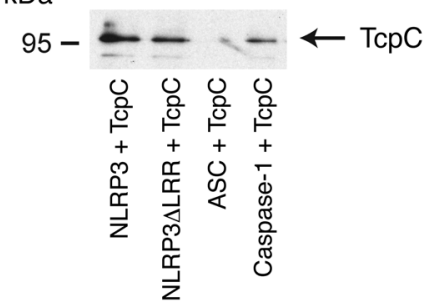

G IP anti-FLAG

kDa WB anti-FLAG

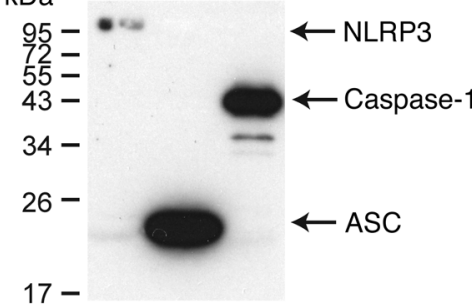

H IP anti-FLAG

$\mathrm{kDa} \quad$ WB anti-EGFP

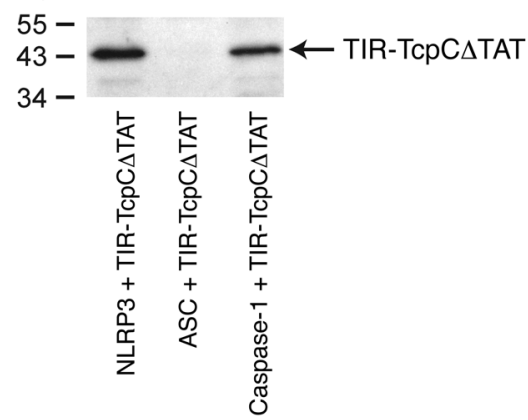

I

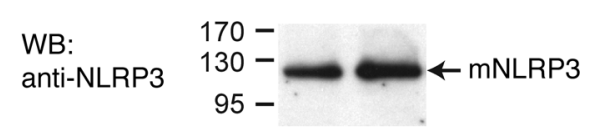

WB: anti-strep

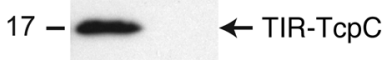

IP: anti-strep $170-$

WB:

anti-NLRP3

BMM lysate TIR-TcpC-strep

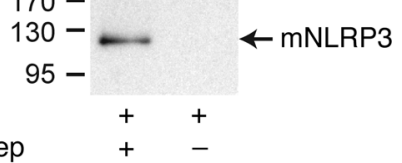

J

WB: $\quad 170-$

anti-NLRP3 $130-$

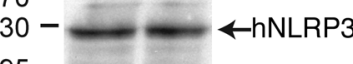

WB:

anti-strep

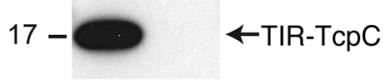

IP: anti-strep

WB:

anti-NLRP3

HCV29 lysate

TIR-TcpC-strep

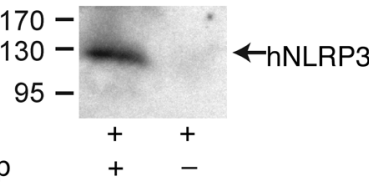

K $\quad \mathrm{kDa}$

WB: anti-ASC $25-\longrightarrow \longrightarrow$ mASC

$\begin{array}{ll}\text { WB: } & 55-\simeq \longrightarrow \text { mCaspase-1 } \\ \text { anti-Caspase-1 } & 40-\end{array}$

WB:

anti-strep

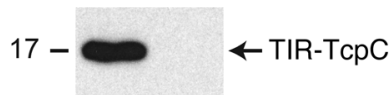

IP: anti-strep

WB:

anti-ASC

IP: anti-strep

WB:

anti-Caspase-1

BMM lysate

TIR-TcpC-strep

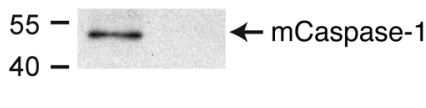

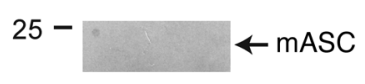

Figure 4. TcpC bound via its TIR domain to NLRP3 and caspase-1, but not to ASC. (A-D) We transfected HEK293 cells with EGFP/DsRed double-labeled TcpC together with FLAG-tagged NLRP3, FLAG-tagged NLRP3ALRR, FLAG-tagged ASC, or FLAG-tagged caspase-1. Transiently transfected cells were lysed after 48 hours, and the lysate was subsequently immunoprecipitated with anti-FLAG affinity beads. (A and B) Expression of TcpC and inflammasome proteins in the cell lysates, (C) enriched FLAG-labeled proteins, (D) amount of precipitated TcpC. Bound TcpC was visualized using anti-EGFP antibodies. (E-H) We replaced EGFP/DsRed double-labeled TcpC with EGFP-labeled TIR-TcpC $\Delta$ TAT for cotransfection of HEK293 cells together with FLAG-tagged NLRP3, FLAG-tagged caspase-1, or FLAG-tagged ASC and immunoprecipitated FLAG-labeled proteins with anti-FLAG affinity beads. In addition, we performed pulldown assays with cell lysates of LPS-stimulated BMDMs. Recombinant purified Strep-tagged TIR-TcpC was immobilized to agarose-coupled streptavidin and incubated with BMDM lysate. Bound NLRP3 was visualized using an anti-NLRP3 antibody (I). We repeated the experiment and replaced BMDMs with the human uroepithelial cell line HCV29 to explore whether human NLRP3 also interacts with TIR-TcpC (J). We also used BMDM lysates to detect whether ASC or caspase-1 bound to TIR-TcpC. Anti-ASC or anti-caspase-1 antibodies were used to detect bound proteins (K). mCaspase-1, murine caspase-1.

TcpC colocalizes with NLRP3 and caspase-1, but not with ASC. To explore how TcpC modulates the NLRP3 inflammasome, we examined the intracellular colocalization of TcpC with components of the inflammasome using confocal microscopy. We initially failed to detect full-length FLAG-tagged TcpC due to its rapid degradation. We therefore constructed a double-labeled TcpC by addition of EGFP at the $\mathrm{N}$ terminus and DsRed at the $\mathrm{C}$ terminus of the protein to monitor degradation of TcpC by fluorescence microscopy. Interestingly, we were now able to detect the molecule via both fluorescent markers in transfected HEK293 cells, indicating that addition of the fluorescent proteins stabilized it to some extent and allowed the detection of the full-length protein (Figure 3, A-C). Upon cotransfection with components of the NLRP3 inflammasome, we found that TcpC colocalized with NLRP3 (Figure 3A) and caspase-1 
A
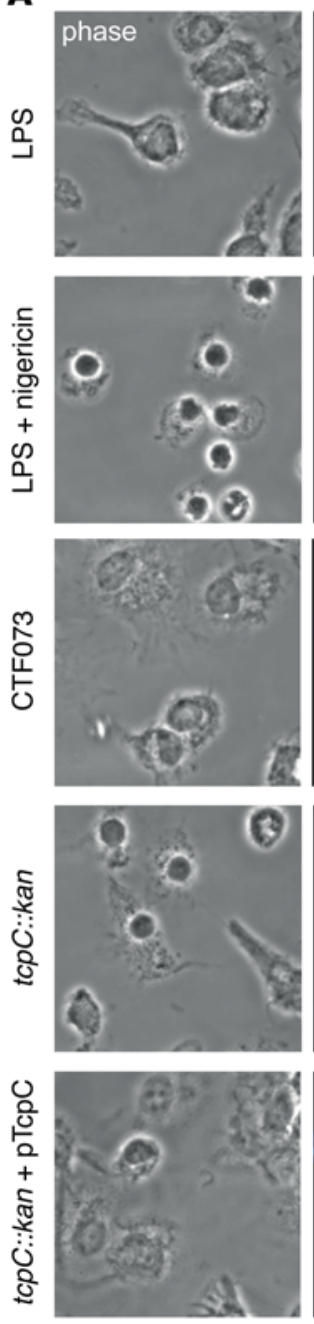
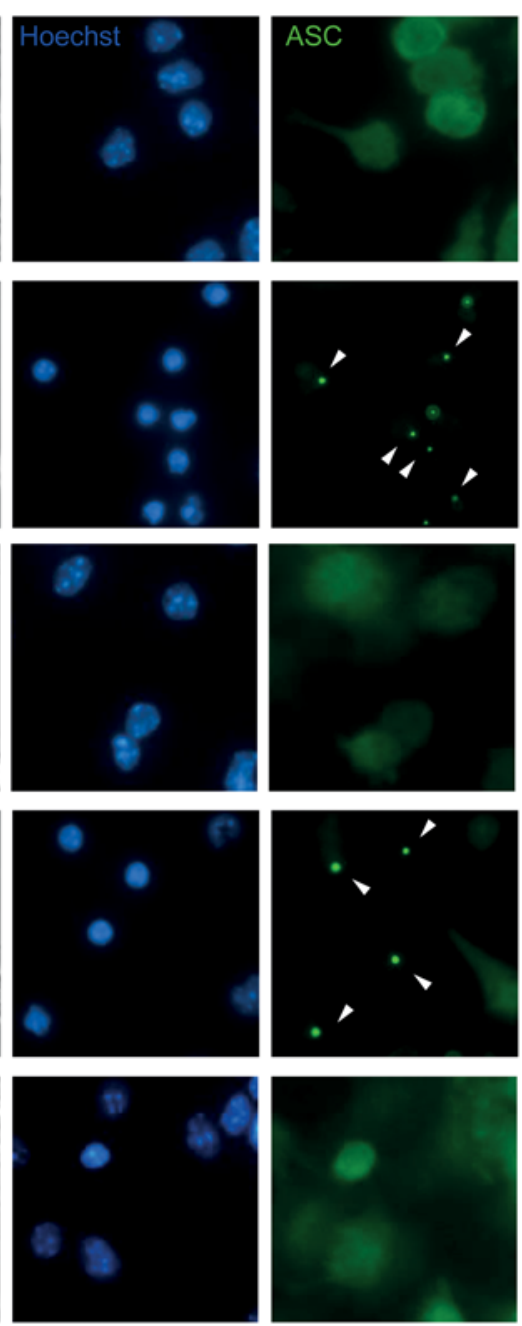
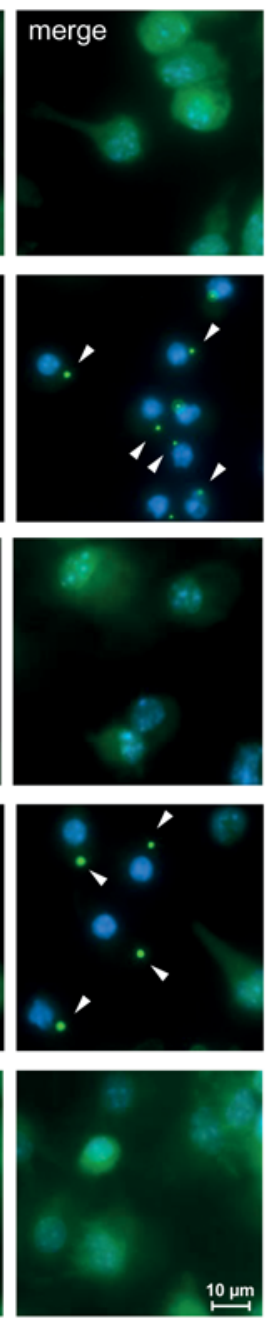

B

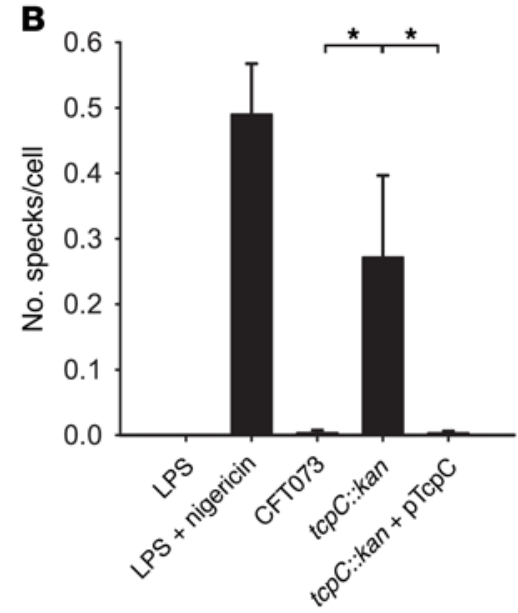

Figure 5. TcpC prevents CFT073-induced speck formation of ASC. (A) We infected LPS-primed BMDCs with CFTO73, the tcpC-deficient CFTO73 strain $t c p C:: k a n$, or the $t c p C$-complemented strain CFT073 tcpC::kan+pTcpC, as indicated (MOI 0.02). LPS served as negative and LPS plus nigericin as positive control. We stained the cells with an anti-ASC antibody and visualized speck formation via fluorescence microscopy (arrowheads). (B) Speck formation was quantified. Error bars represent SD of 6 individual microscopic fields. ${ }^{*} P<0.05$, 1-way ANOVA, post hoc Bonferroni's multiple comparison test.
(Figure 3B), but not with ASC (Figure 3C). A densitometry analysis of the intracellular distributions of TcpC, NLRP3, caspase-1, and ASC showed that these were similar for TcpC, NLRP3, and caspase-1, but not for TcpC and ASC (Supplemental Figure 3, A-C).

We then determined whether the isolated TIR domain of TcpC would also colocalize with NLRP3 and caspase- 1 and transfected HEK293 cells with a plasmid encoding a FLAG-labeled TIR domain of TcpC (TIR-TcpC). This construct was well expressed in HEK293 cells, but to a larger extent, in the nucleus of the cells (data not shown). We observed earlier that a flash-tagged TcpC was found in the cytosol and the nucleus of the host cell upon infection with CFT073 (5). The TIR domain of TcpC colocalized with NLRP3 and caspase-1 (Supplemental Figure 4). In this experiment, the labeling of the colocalizing molecules was reversed in comparison with the previous experiment, i.e., TIR-TcpC was FLAG labeled and NLRP3 and caspase-1 were double labeled with EGFP and DsRed. We think it is highly unlikely that in these 2 experimental settings, EGFP or DsRed was responsible for the colocalization of TcpC with NLRP3 or caspase-1. To optimize the cytosolic expression of the TIR domain of TcpC, we removed the last 15 C-terminal amino acids of TIR-TcpC, which contain 3 arginine residues and 1 lysine and allow the protein to penetrate cellular membranes - similarly to the HIV-TAT protein. Remov- al of this small domain resulted in a protein that we named TIRTcpC $\Delta \mathrm{TAT}$ and that was only expressed in the cytosol (Figure 3, D-F). We used EGFP-labeled TIR-TcpC $\Delta$ TAT and DsRed-labeled NLRP3, caspase-1, or ASC and studied living cells to avoid fixation artifacts. TIR-TcpC $\triangle$ TAT colocalized with NLRP3 (Figure 3D) and caspase-1 (Figure 3E), but not with ASC (Figure 3F). Densitometry again confirmed these results (Supplemental Figure 3, D-F). Thus, the TIR domain of TcpC sufficed for colocalization. When we coexpressed TIR-TcpC $\Delta$ TAT together with NLRP3 and caspase-1, we observed a trimolecular complex, similar to a complex consisting of NLRP3, ASC, and caspase-1 (Figure 3, G and H, and Supplemental Figure 3, G and H). Thus, the TIR domain of TcpC might interact with NLRP3 and caspase-1, forming an alternative complex of the NLRP3 inflammasome.

TcpC interacts with NLRP3 and caspase-1. To demonstrate a direct interaction of TcpC with components of the NLRP3 inflammasome, we performed immunoprecipitation experiments. TcpC was shown to bind to NLRP3 or caspase-1, but not to ASC (Figure $4, \mathrm{~A}-\mathrm{D}$ ). The interaction of TcpC with NLRP3 was independent of its LRR domain, since NLRP3 lacking this domain also precipitated TcpC (Figure 4, A-D). To explore whether the TIR domain of TcpC mediated this interaction, we replaced TcpC with TIRTcpC $\Delta \mathrm{TAT}$ and repeated the experiment. We again found that 
A
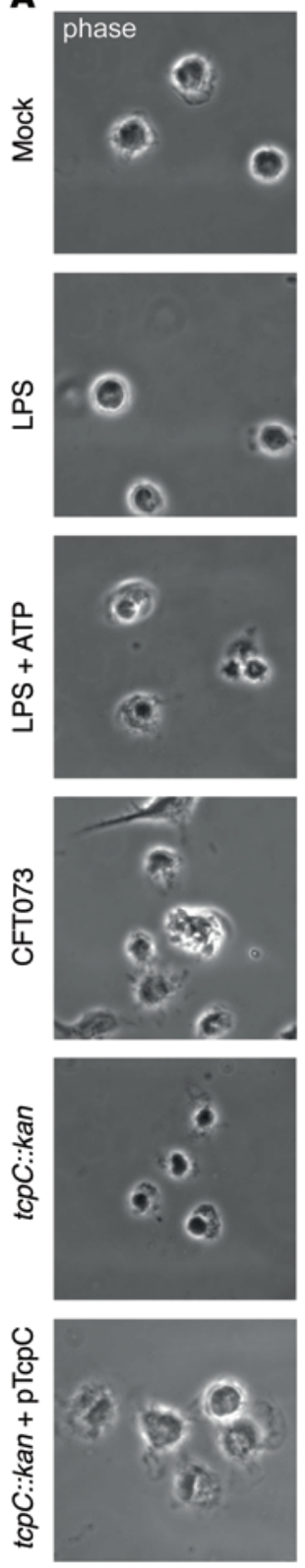
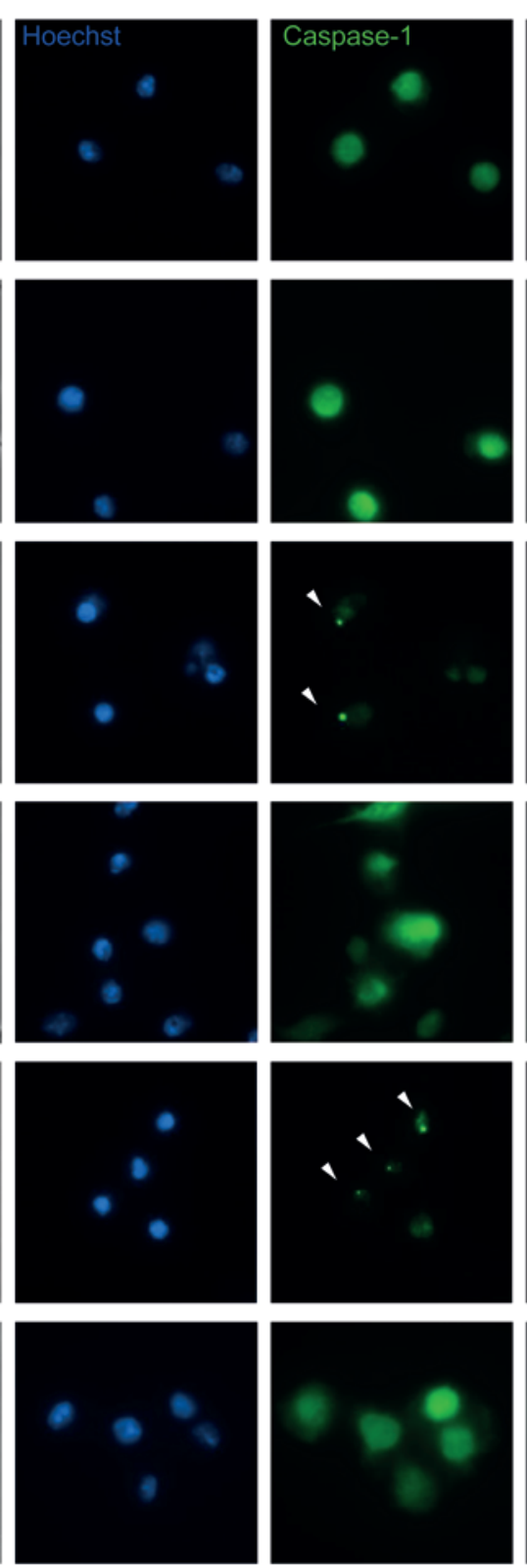
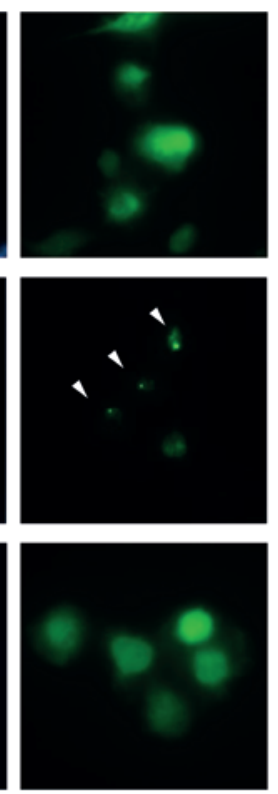
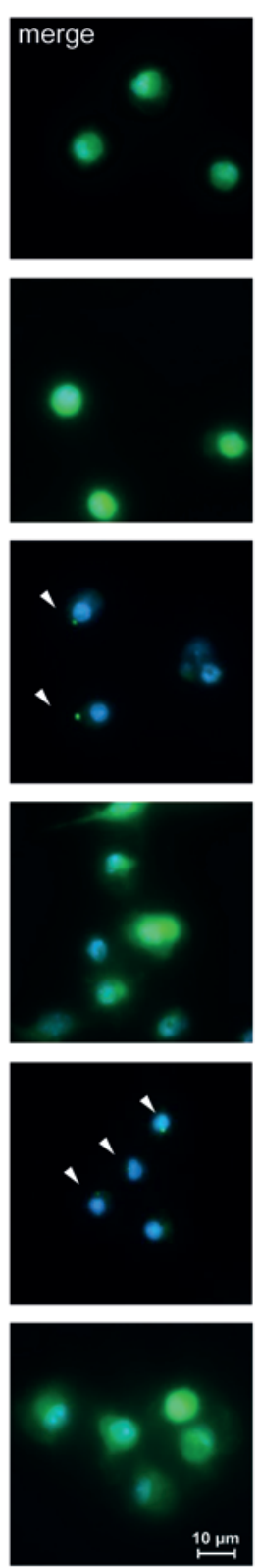

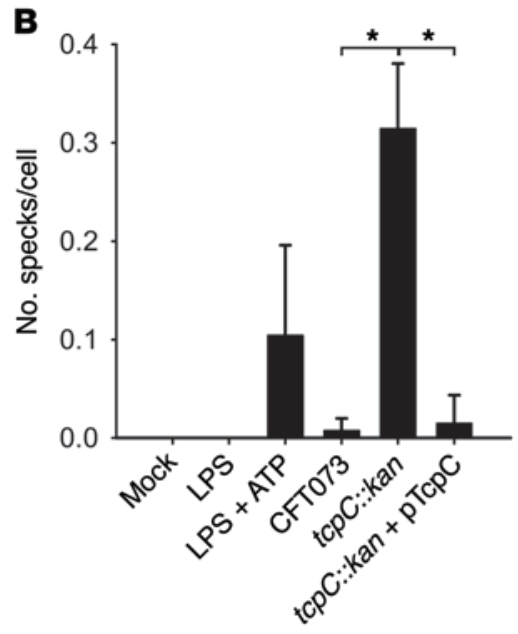

Figure 6. TcpC prevents CFT073-induced speck formation of caspase-1. (A) We infected BMDCs with CFT073, the tcp C-deficient CFT073 tcpC::kan strain or the tcpC-complemented strain CFT073 tcpC::kan+pTcpC as indicated (MOI 0.02). Mock and LPS served as negative and LPS plus ATP as positive control. We stained the cells with an anti-caspase-1 p10 antibody and visualized speck formation via fluorescence microscopy (arrowheads). (B) Speck formation was quantified. Error bars represent SD of 20 individual microscopic fields. The experiments were repeated 3 times with similar results. A representative experiment is shown. ${ }^{*} P<0.05$, 1-way ANOVA, post hoc Bonferroni's multiple comparison test.
TIR-TcpC $\Delta$ TAT was precipitated with NLRP3 and caspase-1, but not with ASC (Figure 4, E-H). Other proteins that were claimed to be involved in the activation of the NLRP3 inflammasome, such as pannexin-1 $(21,22)$, or that belong to other inflammasomes, such as NLRP1, failed to precipitate TIR-TcpC (Supplemental Figure 5). Taken together, these experiments demonstrated that the TIR domain of TcpC interacted with NLRP3 and caspase-1. To further substantiate these findings, we used pull-down assays to demonstrate an interaction of TcpC with endogenous expression levels of NLRP3, caspase-1, or ASC. Using cellular lysates of murine BMDMs or the human epithelial cell line HCV29, we showed that TIR-TcpC bound to murine (Figure 4I) and human NLRP3 (Figure $4 \mathrm{~J}$ ) and murine caspase- 1 (Figure $4 \mathrm{~K}$ ), but not to ASC (Figure $4 \mathrm{~K}$ ) under these more physiological conditions.

TcpC impairs the function of the NLRP3 inflammasome. The data so far show that the presence of the $t c p C$ gene reduced IL- $1 \beta$ secre- tion by CFT073-infected host cells and that TcpC interacted with NLRP3 and caspase-1. To address whether the interaction of TcpC with the NLRP3 inflammasome impairs its function, we reconstituted the NLRP3 inflammasome in HEK293 cells by transfecting the 3 components of the NLRP3 inflammasome, i.e., NLRP3, ASC, and caspase- 1 together with titrated amounts of TcpC. The results showed that overexpression of NLRP3, ASC, and caspase-1 induced the cleavage of the latter molecule (Supplemental Figure $6 \mathrm{~A})$. TcpC was only visible as full-length and cleaved protein at the highest dose used. With increasing amounts of TcpC, the expression of full-length and cleaved caspase- 1 was reduced, while the expression of ASC and NLRP3 remained unaltered or was increased, respectively (Supplemental Figure 6A). Densitometry of the full-length and 20 band of caspase- 1 revealed that the ratio between p20 and full-length caspase- 1 declined dependent on the TcpC dose (Supplemental Figure 6B). 

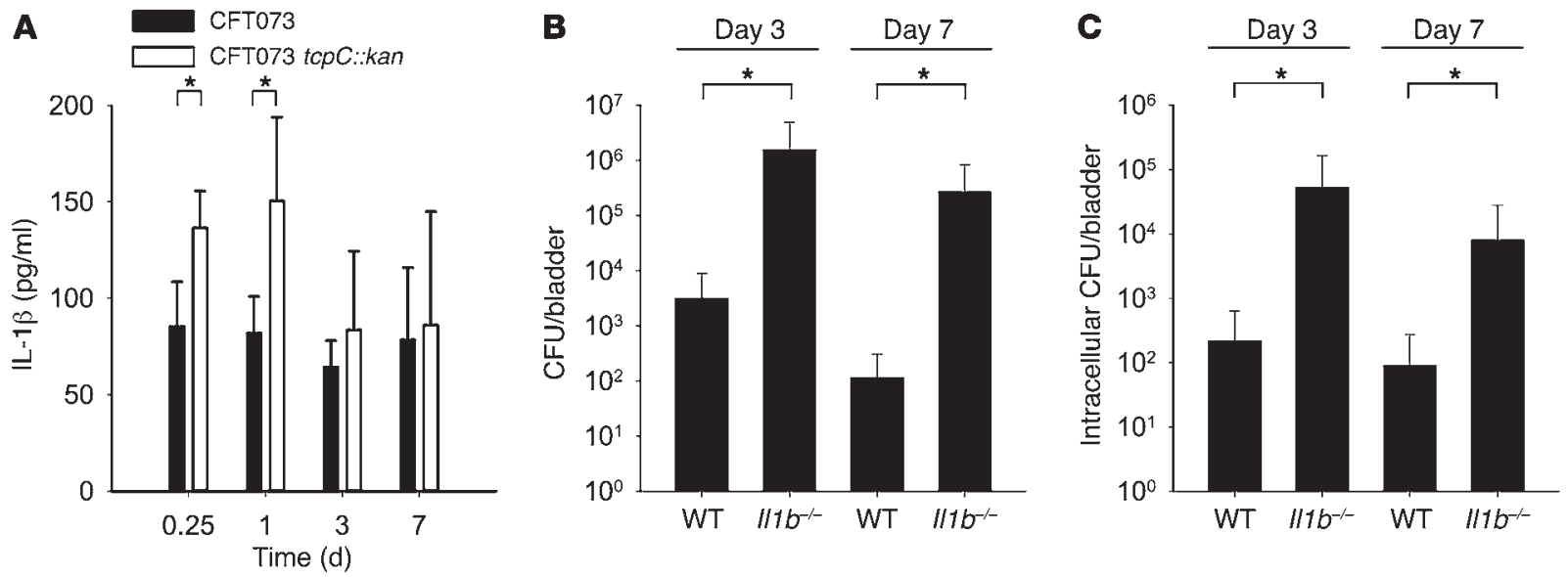

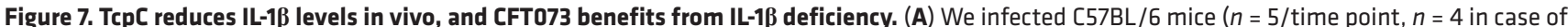
CFT073 infection at day 7) with CFT073 or the tcpC-deficient CFT073 tcpC::kan strain (10 $\left.{ }^{8} \mathrm{CFU} / \mathrm{mouse}\right)$. At the time points indicated after infection, urine IL-1 $\beta$ levels were determined. ${ }^{*} P<0.01, t$ test. Error bars represent SD of 4 or 5 mice. (B) C57BL/ 6 WT or IL-1 $\beta$-deficient mice $(n=7-10)$ were infected with CFT073 $\left(2 \times 10^{5} \mathrm{CFU} /\right.$ mouse) and at the time points indicated, bacterial burden in the bladders was determined. ${ }^{*} P<0.05$, Mann-Whitney rank sum test. (C) We also measured intracellular bacteria of bladder cells of WT and IL-1 $\beta$-deficient mice $(n=7-10) .{ }^{*} P<0.05$, Mann-Whitney rank sum test. Error bars represent SD of 7 to 10 mice (B and $\mathbf{C}$ ).

To extend these results, we analyzed ASC speck formation as a marker of inflammasome activation upon infection of BMDCs with CFT073, the mutant strain CFT073 tcpC::kan, and complemented mutant CFT073 tcpC::kan+pTcpC. While CFT073 tcpC::kan induced ASC speck formation similarly to the positive control stimulation of LPS plus nigericin, the WT strain CFT073 and the complemented mutant failed to do so (Figure 5). When we analyzed the formation of caspase- 1 foci, CFT073 again prevented the speckled formation of caspase-1, while the mutant strain CFT073 tcpC::kan induced caspase-1 specks (Figure 6).

These findings suggest that $\mathrm{TcpC}$ prevented speck formation of ASC and caspase- 1 and consequently reduced the capacity of the NLRP3 inflammasome to process caspase-1 into its proteolytically active form.

$T c p C$ reduced IL-1 $\beta$ levels in the urine of CFTO73-infected mice. To determine whether TcpC influenced IL-1 $\beta$ secretion in response to $\mathrm{UTI}$, mice were infected with $\mathrm{CFT} 073$ or the $t c p C$-deficient mutant CFT073 tcpC::kan. Urine IL-1 $\beta$ levels were determined at different time points after infection. The results revealed that CFT073infected mice displayed reduced IL-1 $1 \beta$ levels at 6 and 24 hours after infection compared with mice infected with CFT073 tcpC::kan (Figure 7A), although CFT073 replicated more efficiently in vivo than CFT073 tcpC::kan $(5,13)$. At later time points, no difference was observed. We then infected IL-1 $\beta$-deficient mice with CFT073 to explore whether a deficiency of the cytokine would influence the bacterial load. We found, indeed, that the bladders of IL-1 $\beta$-deficient mice harbored about 2 to 3 orders of magnitude higher numbers of bacteria than those of WT mice (Figure 7B). We obtained almost identical results when we analyzed the number of intracellular bacteria (Figure 7C). These results show that a reduction of IL-1 $\beta$ secretion by TcpC was beneficial for the bacterium during UTI.

\section{Discussion}

Enterobacteriaceae, such as E. coli, Citrobacter rodentium, or Salmonella enterica ssp. enterica serovar Typhimurium, have been reported to stimulate a noncanonical pathway of the NLRP3 inflam- masome (20, 23), which requires NLRP3, ASC, and caspase- 1 and, in addition, caspase- 11 for IL-1 $\beta$ secretion, while NLRP3, ASC, and caspase-1 (but not caspase-11) are involved in the canonical pathway, triggered, for example, by the combination of LPS and ATP. While caspase- 11 was sufficient to mediate pyroptosis and release of IL- $1 \alpha$, secretion of mature IL- $1 \beta$ and IL-18 depended additionally on caspase-1 $(20,24)$. CFT073 induced IL-1 $\beta$ secretion via NLRP3, ASC, and caspase-1. Since we used double-deficient caspase-1/11 mice we can neither exclude nor confirm a role for caspase-11 in the recognition of this pathogen. However, caspase-1 is crucial for IL-1 $\beta$ release during an infection with CFT073.

Several mechanisms were developed by Enterobacteriaceae to reduce recognition by inflammasomes. Yersinia induced IL-1 $\beta$ secretion via NLRP3 and the NOD-like receptor family caspase activation and recruitment domain-containing (CARD-containing) protein 4 (NLRC4), recognizing components of the type III secretion system (T3SS) (25). This response was impaired by YopK, which interacted with the T3SS and prevented inflammasome activation. Moreover, Yersinia translocated YopM via its T3SS into the cytosol of host cells, which impaired IL-1 $\beta$ secretion by binding to caspase-1 (26). Intracellular Shigella remodeled their highly stimulatory endotoxin to a weakly stimulatory form, thereby reducing the inflammasome-mediated secretion of IL-1 $\beta$ (27). Moreover, Salmonella impaired the expression of NLRC4 in B cells, which prevented IL-1 $\beta$ secretion (28). These different bacterial inflammasome subversion strategies underline the relevance of this system for the antibacterial host defense. In contrast to Yersinia and Salmonella, CFT073 appeared to induce IL-1 $1 \beta$ secretion exclusively via the NLRP3 inflammasome. Our findings identify $\mathrm{TcpC}$ as a new and potent inflammasome inhibitor reducing bacterial recognition by this host defense machinery.

TcpC targets central components of innate immune pathways. Since caspase-1 is activated by all inflammasomes to mature IL-1 $\beta$ (16), its inactivation is therefore highly effective for switching off a major part of intracellular pathogen-recognition receptor-mediated (PRR-mediated) effects. Additional bind- 
ing of TcpC to NLRP3, a PRR that senses many different PAMPs and damage-associated molecular patterns (DAMPs) and thus may recognize different components of CFT073, might further increase the efficiency of the microorganism to avoid detection. Recent evidence indicated that absent in melanoma 2 (AIM2) and NLRP3 inflammasomes were activated by 2 polymerization processes. First, activated AIM2 or NLRP3 induces the polymerization of the pyrin domain of ASC. This process in turn precipitates filaments of the CARD of caspase- 1 and, as a consequence, the enzyme is activated $(29,30)$. Thus, in this new model, AIM2 or NLRP3 induced ASC filaments that in turn are in the center of caspase- 1 filaments. The interaction of TcpC with NLRP3 and caspase-1 might therefore be more efficient to prevent the formation of these NLRP3/ASC/caspase-1 complexes.

The strategy of TcpC to interfere twice with an innate recognition system was already observed with its interaction with the TLR system. Here, TcpC bound to MyD88 as the central component of TLR signaling, thereby preventing the activation of the transcription factor NF- $\mathrm{B}$ (5). Aside from TLR3, TLR4 also signals MyD88 independently via TIR domain-containing adaptor protein-inducing IFN- $\beta$ (TRIF). To prevent this, TcpC bound additionally to TLR4 (6). The TIR domain of TcpC was responsible for the binding to MyD88 and also sufficed to interact with NLRP3 and caspase- 1 . While the binding site of TcpC to MyD88 was precisely mapped to amino acids of the CD, DE, and EE loops (6), we demonstrate here that the LRR domain of NLRP3 was dispensable for the interaction with TcpC.

Our results also indicate that $\mathrm{TcpC}$ is an active inhibitor of the NLRP3 inflammasome in vivo. On the one hand, infection with CFT073 increased mucosal expression of NLRP3, ASC, and IL-1 $\beta$ in WT mice. On the other hand, urine IL-1 $\beta$ levels were significantly $(P<0.01)$ reduced upon infection with CFT073 compared with the mutant strain CFT073 tcpC::kan.

The uropathogen-induced apical expression of the NLRP3 inflammasome in the murine bladder epithelium, which recently was also observed in rats (31), points to a role as a first-line recognition system during acute cystitis. Our results also suggest that IL-1 $\beta$ is important for controlling bacterial replication during bladder infection, since the bacterial burden in the urine was increased by several orders of magnitude in IL-1 $\beta$-deficient mice. Thus, reduction of IL- $1 \beta$ levels by TcpC was presumably beneficial for the uropathogen CFT073. This conclusion is supported by the finding that mice deficient for ATG16L1, a key autophagy protein, were protected from UTIs and that this protection depended on IL-1 $\beta$ (32). In vitro ATG16L1-deficient macrophages phagocytized UPECs more efficiently and secreted higher levels of IL-1 $\beta$.

In summary, we show here that the UPEC strain CFT073 elaborated a mechanism for switching off the NLRP3 inflammasome in addition to the TLR system by interacting with central components of this cytosolic recognition system.

\section{Methods}

Mouse strains and murine UTI model. Female WT C57BL/6, NLRP3deficient, ASC-deficient, caspase-1/11-deficient, and IL-1 $\beta$-deficient mice were used at an age of 8 to 16 weeks. IL-1 $\beta$-deficient mice were backcrossed at least 8 times and the remaining gene-deficient mice backcrossed at least 9 times onto the C56BL/6 strain. The $E$. coli strain CFT073 was injected into the urinary tract, as described previously (33). In brief, we instilled $2 \times 10^{5}$ or $2 \times 10^{8} \mathrm{CFU} /$ mouse into the bladder of anesthetized mice through a soft polyethylene catheter (0.61 mm outer diameter; Clay Adams, BD). Urine samples were obtained, and the mice were sacrificed at the indicated time period after infection. Bacterial tissue counts were obtained after homogenization and plating. The number of intracellular bacteria was determined by killing extracellular bacteria with gentamycin $(200 \mu \mathrm{g} / \mathrm{ml})$ and subsequent washing and homogenization of the remaining organ.

Cell lines and generation of BMDMs or BMDCs. HEK293 cells were obtained from ATCC. The human uroepithelial cell line HCV29 was provided by Sören Schubert (34).

Murine BM was prepared from femora and tibiae of WT C57BL/6, NLRP3-deficient, ASC-deficient, and caspase-1/11-deficient mice and was rinsed with cell culture medium.

BMDMs were cultured on bacteriologic petri dishes overnight in culture medium (DMEM, 10\% heat-inactivated FCS, $100 \mathrm{IU} / \mathrm{ml}$ penicillin, $100 \mathrm{mg} / \mathrm{ml}$ streptomycin [PAA Laboratories], and 50 mM 2-ME [Invitrogen]) in the presence of L cell-conditioned medium (LCCM) as a source of macrophage CSF (M-CSF) $(15 \% \mathrm{v} / \mathrm{v})$ to remove adherent cells. Nonadherent cells were plated at a density of $8 \times 10^{6}$ cells/dish and cultivated for another 6 days in complete medium in the presence of LCCM $(15 \% \mathrm{v} / \mathrm{v})$, which was added a second time on day 3. Cells were washed vigorously, and only adherent macrophages were used 7 days after plating.

Conventional BMDCs were prepared from femora and tibiae of mice, as described for BMDMs. They were plated on bacterial petri dishes overnight in culture medium (RPMI 1640, 10\% heat-inactivated FCS, $100 \mathrm{IU} / \mathrm{ml}$ penicillin, $100 \mathrm{mg} / \mathrm{ml}$ streptomycin [PAA Laboratories], and $50 \mathrm{mM}$ 2-ME [Invitrogen]) in the presence of $20 \mathrm{ng} / \mathrm{ml}$ granulocytemacrophage CSF (GM-CSF) (Peprotech) at a density of $6 \times 10^{6}$ cells/dish and cultivated for another 6 days in complete medium in the presence of $20 \mathrm{ng} / \mathrm{ml} \mathrm{GM-CSF}$, which was added a second time on day 3 .

Antibodies used for Western blots and cytokine ELISAs. The antibodies specific for MYC and FLAG epitopes were provided by SigmaAldrich, anti-GFP was from Cell Signaling Technology, antibodies specific for NLRP3 and the p20-domain of caspase-1 (clone Casper-1) were purchased from AdipoGen, and the antibody specific for the p10-domain (M-20) came from Santa Cruz Biotechnology Inc. Antibodies specific for ASC or IL-1 $\beta$ came from Merck Millipore or R\&D Systems, respectively. The secondary antibodies anti-rabbit HRP and anti-mouse HRP were from Jackson ImmunoResearch, anti-goat HRP was from Promega, and anti-rabbit Alexa Fluor 488 was from Life Technologies (Thermo Fisher). IL-1 $\beta$ was quantified in culture supernatants using an ELISA Duo set (R\&D Systems) as described by the manufacturer. LDH was determined in cell culture supernatants using the CytoTox 96 Non-Radioactive Cytotoxicity Assay Kit (Promega). The assay was performed as described by the manufacturer.

Immunohistochemistry. For immunohistochemistry, bladders from 5 mice per experimental group were frozen, cryosectioned, and stained with antibodies against neutrophils (Abcam, ab2557), E. coli (Novus Biologicals, NB200-579), NLRP3 (Santa Cruz Biotechnology Inc., sc66846), IL-1 $\beta$ (Abcam, ab9722), or ASC (Abcam, ab155970). Secondary antibodies were labeled with Alexa Fluor 488 or Alexa Fluor 568, and DAPI was used as a nuclear stain. Sections were exam- 
ined by fluorescence microscopy (Carl Zeiss) with an AxioCam ERc 5s camera at $\times 200$ magnification.

Reagents. ATP and nigericin were bought from Sigma-Aldrich and LPS O111:B4 Ultrapure was from InvivoGen. The caspase inhibitor IV (Z-YVAD-FMK), which impairs caspase-1, was purchased from Merck Millipore (Calbiochem). Anti-FLAG M2 affinity gel came from Sigma-Aldrich.

Plasmids. We cloned full-length TcpC, TIR-TcpC, and TIRTcpC $\triangle \mathrm{TAT}$ into the eukaryotic expression vector pBEN (gift from Frank Schmitz, Seattle Biomedical Research Institute, Seattle, Washington, USA), which contained EGFP alone or EGFP in combination with DsRed and confirmed integrity by sequencing. Human NLRP3, human NLRP3 lacking the LRR domain, human NLRP1, human ASC, human caspase-1, and human pannexin-1 were also subcloned into pBEN, which encoded for a FLAG or MYC label.

E. coli strains. The UPEC strain CFTO73 was provided by ATCC. The tcpC-deficient CFT073 tcpC::kan mutant strain was constructed as described (35). CFT073 tcpC::kan was complemented with the plasmid pTcpC, as described previously (5).

Confocal microscopy. We transiently transfected HEK293 cells with full-length TcpC, TIR-TcpC, TIR-TcpC $\Delta$ TAT, NLRP3, caspase-1, and ASC in various combinations, as indicated in the individual experiments. Transfection was performed using Lipofectamine 2000 (Invitrogen) according to the manufacturer's instructions. The amount of DNA used for transfection was plasmid dependent and varied between 0.3 and $2 \mu \mathrm{g} / \mathrm{ml}$. HEK293 cells were cultured for 48 hours after transfection. The total amount of transfected DNA was kept constant by the addition of an empty vector. Subsequently, we fixed the cells with $2 \%$ paraformaldehyde. Cells were analyzed using a Leica SP5 instrument (Leica Microsystems). In some experiments, living cells were analyzed.

Infection and stimulation assays. BMDMs, BMDCs, and HCV29 cells were seeded in 6- or 12-well plates at concentrations between 1.0 and $2.0 \times 10^{6} /$ well in DMEM or RPMI 1640 supplemented with $10 \% \mathrm{FCS}, 100 \mathrm{IU} / \mathrm{ml}$ penicillin, $100 \mathrm{mg} / \mathrm{ml}$ streptomycin, and $50 \mathrm{mM}$ 2-ME. Prior to each assay, cells were washed and new antibiotic-free medium (3\%-5\% FCS) was added. Cells were then either infected with varying amounts of bacteria as indicated or were stimulated with endotoxin $(20 \mathrm{ng} / \mathrm{ml})$ for 3 hours. Endotoxin-stimulated cells were pulsed with ATP $(5 \mathrm{mM})$ for 30 minutes at the end of the culture period. Culture supernatants were used for quantification of IL-1 $1 \beta$ by ELISA or analysis by Western blotting.

Immunofluorescence. Caspase- 1 formation as a marker for inflammasome activation was determined by using the inhibitor Z-YVADFMK (10 $\mu \mathrm{M}$, Calbiochem) 1 hour prior to infection or stimulation with LPS (20 ng/ml) and ATP (5 mM) of BMDCs on glass coverslips (Lab-Tek chamber slide system, Nunc). Immunofluorescent staining for ASC foci formation was performed with LPS-primed $(20 \mathrm{ng} / \mathrm{ml})$ BMDCs before infection or stimulation with nigericin $(5 \mu \mathrm{M})$ on glass coverslips. BMDCs were washed with PBS and fixed in $4 \%$ paraformaldehyde for 15 minutes and permeabilized using 0.1\% Triton X-100 (Sigma-Aldrich) for 10 minutes. Cells were stained with rabbit anticaspase- 1 antibody (1:100, sc 514; Santa Cruz Biotechnology Inc.) or anti-ASC antibody (1:400, AL177, Adipogen) followed by a secondary Alexa Fluor 488-labeled antibody (1:400, Life Technologies) in combination with Hoechst 33342 (1:10000, Life Technologies). Coverslips mounted with Roti-Mount FluorCare (Carl Roth) were examined by fluorescence microscopy (Leica DMRBE). Caspase-1 or ASC foci formation was enumerated by counting at least 250 cells in each experiment at $\times 40$ or $\times 100$ magnification.

Pull-down experiments. Purified TIR-TcpC carrying a C-terminal Strep-tag II was used as "bait." $80 \mu$ g of purified TIR-TcpC was bound to $50 \%(\mathrm{v} / \mathrm{v})$ suspension of Strep-Tactin MacroPrep Beads (IBA Lifesciences) in buffer PD1 (100 mM Tris· $\mathrm{HCl}, \mathrm{pH}$ 8.0, $1 \mathrm{mM}$ EDTA, and $150 \mathrm{mM} \mathrm{NaCl}$ ) for 1.5 hours at $4^{\circ} \mathrm{C}$ under gentle agitation. After 3 washing steps and 1 blocking step with avidin $(300 \mu \mathrm{g} /$ $\mathrm{ml}$ in PD1) at room temperature with occasional rocking, 50 to 200 mg cleared total cell lysates ("prey") from BMDMs or HCV29 cells were added and incubated for 2 hours at $4^{\circ} \mathrm{C}$, followed by $30 \mathrm{~min}$ utes at $37^{\circ} \mathrm{C}$. The beads were then washed 3 times with an acetate buffer (catalog 1858605, Pierce/Thermo Scientific) containing 100 $\mathrm{mM}$ or $500 \mathrm{mM} \mathrm{NaCl}$ to remove nonspecifically bound proteins. The bound prey proteins were then eluted in 2 consecutive steps with an elution buffer (100 mM sodium citrate, $100 \mathrm{mM}$ glycine, and $3 \mathrm{mM}$ EDTA) at $\mathrm{pH} 2.8$ and neutralized with $2 \mathrm{M}$ Tris $\cdot \mathrm{HCl}(\mathrm{pH}$ 8.0). Samples were then stored at $-20^{\circ} \mathrm{C}$ before analysis by SDSPAGE or Western blot.

To obtain the cell lysates for pull-down analysis, $5 \times 10^{6}$ cells were stimulated with $100 \mathrm{ng} / \mathrm{ml}$ LPS for 24 hours and then solubilized in Nonidet P-40 lysis buffer containing 50 mM Hepes-Na (pH 7.6), 150 mM NaCl, 1 mM DTT, 1 mM EDTA, 1 mM EGTA, 0.5\% (v/v) Nonident P-40, 0.125\% (w/v) N-octyl- $\beta$-D-glucopyranoside, $10 \%$ (v/v) glycerol, $20 \mathrm{mM} \beta$-glycerolphosphate, $1 \mathrm{mM} \mathrm{Na} \mathrm{VO}_{4}, 0.4 \mathrm{mM} \mathrm{PMSF}$, and 1 $\mathrm{mM} \mathrm{NaF}$, plus the cOmplete Protease Inhibitor mixture (EDTA-Free, Roche Diagnostics). Lysates were subsequently homogenized using a tissue grinder and dialyzed against PBS plus the protease inhibitor.

Coimmunoprecipitation and Western blot. Transiently transfected cells using Lipofectamine 2000 (Invitrogen) according to the manufacturer's instructions were lysed 48 hours after transfection with Nonidet P-40 lysis puffer as described for pull-down experiments and immunoprecipitated with anti-FLAG beads overnight at $4^{\circ} \mathrm{C}$ on a rotating incubator. Beads were washed 3 times with PBS containing $0.5 \mathrm{M} \mathrm{NaCl}$ before bound immunoprecipitates were eluted by incubation with $20 \mu \mathrm{l} 1 \times$ SDS sample buffer (62.5 mM Tris, 6,24\% glycerol, $2 \%$ SDS, $25 \mathrm{mM} \mathrm{DTT}$ ) at $95^{\circ} \mathrm{C}$ for 5 minutes and visualized by Western blot.

Cells from infection and stimulation assays were washed once with PBS, lysed in $1 \times$ SDS sample buffer, and subjected to Western blot analysis for the presence of pro-IL-1 $\beta$, pro-caspase-1, and inflammasome components.

All immunoblotting probes were separated by $10 \%, 12 \%$, or $15 \%$ PAGE and transferred to a PVDF membrane. Membranes were incubated with anti-IL-1 $\beta$ (diluted 1:1.000), anti-NLRP3 (diluted 1:1.000), anti-caspase-1 p20 (diluted 1:1.000), anti-ASC (1:1.000), anti- $\beta$-actin (diluted 1:10.000), anti-MYC (diluted 1:1.000), antiFLAG (diluted 1:1.000), or anti-EGFP (diluted 1:1.000). All primary antibodies except for ASC (TBST with 1\%BSA) were diluted in TBST (100 mM Tris $\cdot \mathrm{HCl}, \mathrm{pH} 7.6,150 \mathrm{mM} \mathrm{NaCl}$, and 0.05\% Tween-20 containing $5 \%$ milk powder) and used overnight at $4{ }^{\circ} \mathrm{C}$. After 3 washing steps with TBST, the secondary antibodies were added (diluted 1:5,000 in TBST containing 5\% milk powder, 1 hour, room temperature). The blot was washed 3 times with TBST and visualized using the Western Lightning Plus chemiluminescence reagent (PerkinElmer) as described by the manufacturer. 
Statistics. Statistics were performed using Prism 5 (GraphPad software). One-way ANOVA and Bonferroni's multiple comparison test as post hoc were used to compare more than 2 groups for statistical differences; $t$ test or Mann-Whitney rank sum test was taken to compare 2 groups. $P$ values of less than 0.05 were considered significant.

Study approval. All experiments involving animals were performed with the permission of the Animal Experimental Ethics Committee at the Lund District Court, Lund, Sweden (numbers M44-13).

\section{Author contributions}

A Waldhuber performed most experiments, and collected and analyzed data. MP, YZ, and A Wieser performed in vivo experiments. CC, SD, SA, SNP, FR, and T Müller performed experiments in vitro. SS, OG, and CS designed experiments, discussed the data, and contributed to the writing of the manuscript. T Miethke conceived and designed the study and wrote the manuscript.

\section{Acknowledgments}

This work was supported by Deutsche Forschungsgemeinschaft (DFG) grant MI471/6-1.

Address correspondence to: Thomas Miethke, Institute of Medical Microbiology and Hygiene, University of Heidelberg, Theodor-Kutzer-Ufer 1-3, 68167 Mannheim, Germany. Phone:49.621.383.2513;E-mail:Thomas.Miethke@medma.uniheidelberg.de.
1. Foxman B. Epidemiology of urinary tract infections: incidence, morbidity, and economic costs. Dis Mon. 2003;49(2):53-70.

2. Ulett GC, Totsika M, Schaale K, Carey AJ, Sweet MJ, Schembri MA. Uropathogenic Escherichia coli virulence and innate immune responses during urinary tract infection. Curr Opin Microbiol. 2013;16(1):100-107.

3. Zhou G, et al. Uroplakin Ia is the urothelial receptor for uropathogenic Escherichia coli: evidence from in vitro FimH binding. J Cell Sci. 2001;114(pt 22):4095-4103.

4. Dodson KW, Jacob-Dubuisson F, Striker RT, Hultgren SJ. Outer-membrane PapC molecular usher discriminately recognizes periplasmic chaperone-pilus subunit complexes. Proc Natl Acad Sci U S A. 1993;90(8):3670-3674.

5. Cirl C, et al. Subversion of Toll-like receptor signaling by a unique family of bacterial Toll/interleukin-1 receptor domain-containing proteins. Nat Med. 2008;14(4):399-406.

6. Snyder GA, et al. Molecular mechanisms for the subversion of MyD88 signaling by TcpC from virulent uropathogenic Escherichia coli. Proc Natl Acad Sci U S A. 2013;110(17):6985-6990.

7. Salcedo SP, et al. Brucella control of dendritic cell maturation is dependent on the TIR-containing protein Btp1. PLoS Pathog. 2008;4(2):e21.

8. Newman RM, Salunkhe P, Godzik A, Reed JC. Identification and characterization of a novel bacterial virulence factor that shares homology with mammalian Toll/interleukin-1 receptor family proteins. Infect Immun 2006;74(1):594-601.

9. Low LY, Mukasa T, Reed JC, Pascual J. Characterization of a TIR-like protein from Paracoccus denitrificans. Biochem Biophys Res Commun. 2007;356(2):481-486.

10. Snyder GA, et al. Crystal structures of the Toll/ Interleukin-1 receptor (TIR) domains from the Brucella protein TcpB and host adaptor TIRAP reveal mechanisms of molecular mimicry. J Biol Chem. 2014;289(2):669-679.

11. Welch RA, et al. Extensive mosaic structure revealed by the complete genome sequence of uropathogenic Escherichia coli. Proc Natl Acad Sci US A. 2002;99(26):17020-17024.

12. Schubert $S$, et al. Prevalence and phyloge- netic history of the TcpC virulence determinant in Escherichia coli. Int J Med Microbiol. 2010;300(7):429-434.

13. Yadav M, et al. Inhibition of TIR domain signaling by TcpC: MyD88-dependent and independent effects on Escherichia coli virulence. PLOS Pathog. 2010;6(9):e1001120.

14. Wright KJ, Seed PC, Hultgren SJ. Development of intracellular bacterial communities of uropathogenic Escherichia coli depends on type 1 pili. Cell Microbiol. 2007;9(9):2230-2241.

15. Chen SL, et al. Positive selection identifies an in vivo role for FimH during urinary tract infection in addition to mannose binding. Proc Natl Acad Sci U S A. 2009;106(52):22439-22444.

16. Schroder K, Tschopp J. The inflammasomes. Cell. 2010;140(6):821-832.

17. Schaale K, et al. Strain- and host species-specific inflammasome activation, IL- $1 \beta$ release, and cell death in macrophages infected with uropathogenic Escherichia coli. Mucosal Immunol. 2016;9(1):124-136.

18. Greten FR, et al. NF- $\kappa \mathrm{B}$ is a negative regulator of IL- $1 \beta$ secretion as revealed by genetic and pharmacological inhibition of IKKbeta. Cell. 2007;130(5):918-931.

19. Unlu S, et al. Phosphorylation of IRF8 in a pre-associated complex with Spi-1/PU.1 and non-phosphorylated Stat1 is critical for LPS induction of the IL1B gene. Mol Immunol. 2007;44(13):3364-3379.

20. Kayagaki N, et al. Non-canonical inflammasome activation targets caspase-11. Nature. 2011;479(7371):117-121.

21. Kanneganti TD, et al. Pannexin-1-mediated recognition of bacterial molecules activates the cryopyrin inflammasome independent of Toll-like receptor signaling. Immunity. 2007;26(4):433-443.

22. Qu Y, et al. Pannexin-1 is required for ATP release during apoptosis but not for inflammasome activation. J Immunol. 2011;186(11):6553-6561.

23. Broz P, et al. Caspase-11 increases susceptibility to Salmonella infection in the absence of caspase-1. Nature. 2012;490(7419):288-291.

24. Rathinam VA, et al. TRIF licenses caspase11-dependent NLRP3 inflammasome activation by gram-negative bacteria. Cell.
2012;150(3):606-619.

25. Brodsky IE, et al. A Yersinia effector protein promotes virulence by preventing inflammasome recognition of the type III secretion system. Cell Host Microbe. 2010;7(5):376-387.

26. LaRock CN, Cookson BT. The Yersinia virulence effector YopM binds caspase-1 to arrest inflammasome assembly and processing. Cell Host Microbe. 2012;12(6):799-805.

27. Paciello I, et al. Intracellular Shigella remodels its LPS to dampen the innate immune recognition and evade inflammasome activation. Proc Natl Acad Sci U S A. 2013;110(46):E4345-E4354.

28. Perez-Lopez A, Rosales-Reyes R, AlpucheAranda CM, Ortiz-Navarrete V. Salmonella downregulates Nod-like receptor family CARD domain containing protein 4 expression to promote its survival in B cells by preventing inflammasome activation and cell death. J Immunol. 2013;190(3):1201-1209.

29. $\mathrm{Lu} \mathrm{A}$, et al. Unified polymerization mechanism for the assembly of ASC-dependent inflammasomes. Cell. 2014;156(6):1193-1206.

30. Cai X, et al. Prion-like polymerization underlies signal transduction in antiviral immune defense and inflammasome activation. Cell. 2014;156(6):1207-1222.

31. Hughes FM Jr, et al. Inflammasomes are important mediators of cyclophosphamide-induced bladder inflammation. Am J Physiol Renal Physiol. 2014;306(3):F299-F308.

32. Symington JW, et al. ATG16L1 deficiency in macrophages drives clearance of uropathogenic E. coli in an IL-1 $\beta$-dependent manner. Mucosal Immunol. 2015;8(6):1388-1399.

33. Hagberg L, Hull R, Hull S, Falkow S, Freter R, Svanborg Edén C. Contribution of adhesion to bacterial persistence in the mouse urinary tract. Infect Immun. 1983;40(1):265-272.

34. Bean MA, Pees H, Fogh JE, Grabstald H, Oettgen HF. Cytotoxicity of lymphocytes from patients with cancer of the urinary bladder: detection by a 3-H-proline microcytotoxicity test. Int J Cancer. 1974;14(2):186-197.

35. Datsenko KA, Wanner BL. One-step inactivation of chromosomal genes in Escherichia coli $\mathrm{K}-12$ using PCR products. Proc Natl Acad Sci U S A. 2000;97(12):6640-6645. 